es sich um ein stark kompetitives Feld mit einem bedeutenden öffentlichen Auftrag handelt, wird die Frage nach antirassistischen Strategien in der Medienproduktion so nicht beantwortet. Radikale Kritik ist in diesem Forschungsfeld erforderlich - nicht zuletzt aufgrund des vielbesungenen Mantras, Journalismus sei ein gerechter Ort, in dem nur Professionalität zähle.

Assimina Gouma

\section{Klaus Arnold / Christoph Classen / Susanne Kinnebrock / Edgar Lersch / Hans-Ulrich Wagner (Hrsg.) \\ Von der Politisierung der Medien zur Medialisierung des Politischen?}

Zum Verhältnis von Medien, Öffentlichkeiten und Politik im 20. Jahrhundert

Leipzig: Leipziger Universitätsverlag 2010. $471 \mathrm{~S}$.

\section{ISBN 978-3-86583-497-3}

Medialisierung auch der Politik ist eines der „Trendthemen“ in den Sozialwissenschaften und insbesondere in der Kommunikations- und Medienwissenschaft. Ein sich änderndes Verhältnis von Politik und Medien, eine zunehmende Prägung des politischen Betriebs und der Gestaltung von Politik durch die Massenmedien, ihre Anpassung an die Darstellungslogiken des Vermittlungssystems, an die Erwartungen auch der Journalisten wie der Rezipienten - mit möglichen Tendenzen wie steigender Visualisierung, Personalisierung, Entertainisierung und Kurzatmigkeit respektive mangelnder Nachhaltigkeit - müsste als Prozess verstanden werden, wird aber vielfach nur als Phänomen beschrieben. Ein Phänomen, das „fast immer [...] nur auf die Gegenwart oder die unmittelbare Vergangenheit des entwickelten Internetbzw. Fernsehzeitalters" (S. 14) bezogen wird, mit einer oftmals kulturkritischen, mit „bisweilen schrille(m) Ton“ (ebd.) vorgebrachten Konnotation, wie Klaus Arnold und Christoph Classen in ihrer eine klare Einordnung bietenden Einleitung zu recht monieren. Mit ihrem opulenten, 18 Aufsätze von Historikern, Politologen und vor allem Kommunikationswissenschaftlern umfassenden Reader wollen sie und ihre Mitherausgeber „das Verhältnis von Medien und Politik mit Hilfe von Fallstudien in einer breiten zeithistorischen Perspektive in den Blick nehmen" (S. 15).

Anders als es der Titel nahelegen könnte, gehen sie nicht davon aus, dass eine einstige Dominanz der Politik über die Medien, eine primäre Steuerung also (auch) der öffentlichen
Kommunikation durch die Regierenden, allmählich abgelöst worden sei eben durch die Medialisierung des Politischen. Vielmehr richten sie den Blick auf Interdependenzen - auf die „gegenseitige Interaktion“ der Sphären Politik und Medien, durch die die „Darstellung und Herstellung von Politik [...] im Laufe des 20. Jahrhunderts zunehmend zusammenzufallen “ (S. 18) scheinen.

Ein erster Block mit drei Beiträgen beschäftigt sich primär mit der Frage, wie man Medialisierung klarer theoretisch erfassen kann, im zweiten und dritten Abschnitt wird dann exemplarisch beleuchtet, wie sich das Verhältnis von Medien und Politik in der ersten, dann der zweiten Hälfte des 20. Jahrhunderts konkret gestaltete, abschließend wird, wiederum in drei Aufsätzen, schlaglichtartig beleuchtet, wie die gegenseitige „Durchdringung" der politischen und der medialen „Sphäre“ früher betrachtet wurde - konkret in der Propagandaforschung, in der Weimarer Republik und durch Vertreter der kritischen Theorie (von Horkheimer bis Habermas).

Einen klaren Bezugsrahmen spannt eingangs Thomas Mergel auf, indem er zunächst (systemtheoretisch) darstellt, wie die „Systeme“ Politik und Medien strukturell gekoppelt sind, dann klarmacht, dass eine völlige Symbiose weder theoretisch denkbar noch praktisch erstrebenswert wäre, würde sie doch zum Funktionsverlust und letztlich zur Auflösung beider Bereiche führen. Weiter plädiert er dafür, „die Eigenlogik der Rezipienten stärker in den Blick zu nehmen und nicht allein aus dem Interesse an Medialisierung auf deren Erfolg zu schließen“ (S. 40).

Manche der Fallbeispiele zeigen dann, dass diese „Eigenlogik“ von Medien und Publikum etwa die Nationalsozialisten geradezu zwang, bei der Ausgestaltung des Radioprogramms auf die Erwartung der Hörer einzugehen (Inge Marszolek), dass sie der Politik- und Regierungspropaganda der Regierung Adenauer in den 50er Jahren enge Grenzen setzte (Norbert Grube), dass ein verändertes Interesse für Politik in den 60er und 70er Jahren Änderungen des Medienangebots und bspw. das Aufkommen alternativer Medien beförderte (Klaus Arnold), dass von der Frühen Neuzeit bis in die Gegenwart hinein in Krisenzeiten das persönliche Gespräch besonders wichtig ist (Rudolf Stöber) und dass generell der Wunsch nach Unterhaltung eine Konstante, ja: der prägende Faktor, der Medienentwicklung ist (Michael Meyen).

Immer wieder bricht sie doch durch, die sehr kritische Analyse des gegenwärtigen „Zustands" - so wenn Thymian Bussemer beklagt: 
„Politik erscheint unter den Bedingungen der beschleunigten Mediengesellschaft mitunter wie ein selbstbezüglicher Kreislauf, in dem Medien Themen aufbauen, Politiker verschiedener Couleur sich für eine Weile im Kielwasser der öffentlichen Erregung mit möglichst radikalen Lösungsvorschlägen überbieten und am Ende entweder ein neues bürokratisches Monstrum geschaffen oder neuerliche Freiheitsbeschränkungen durchgesetzt haben. Die Regierten sehen es und wenden sich $a b$ - um fortan noch intensiver mit Propaganda umworben zu werden" (S. 412). Die Medien aber, so machen Frank Marcinkowski und Adrian Steiner deutlich, „bleiben [...] ,gerufene' Geister und keine diabolische Heimsuchung" (S. 73).

Nicht in allen Aufsätzen steht Medialisierung, stehen das Verhältnis von Politik und Medien und die Interdependenzen der beiden gesellschaftlichen Teilsysteme ganz klar im Fokus. Insgesamt gelingt es in dem sorgfältig edierten und sehr inspirierenden Band aber, das "Trendthema“ solider historisch zu fundieren. Auch bietet er zahlreiche Anknüpfungspunkte für weitere diachrone Forschungsvorhaben, in der das Spannungsverhältnis Medien und Politik - auch über Systemwechsel hinweg - untersucht werden könnte.

Markus Behmer

\author{
Nancy K. Baym \\ Personal Connections in the Digital Age \\ Digital Media and Society Series \\ Cambridge: Polity, 2010. - 183 S. \\ ISBN 978-0-7456-4332-8
}

„What does personal communication mean when it's transmitted through a mass medium?" (3) - mit dieser Frage beschäftigt sich die amerikanische Kommunikationswissenschaftlerin Nancy K. Baym in dem 183-seitigen Buch "Personal Connections in the Digital Age“. Mehr noch greift die Autorin ein überaus aktuelles Thema auf, indem sie fragt, wie und ob sich interpersonale Beziehungen durch die zunehmende Digitalisierung der zwischenmenschlichen Kommunikation verändern. Dafür ist das Buch in sieben Kapitel unterteilt: Nach einer kurzen Einführung benennt Baym diverse Parameter (z. B. Interaktivität, Mobilität), die es möglich machen, digitale Medien voneinander abzugrenzen. Im Anschluss werden Nutzertypen identifiziert und vor dem Hintergrund der Digital Divide-These kritisch reflektiert. Ihre Analyse endet mit der Erkenntnis, dass digitale Medien für unterschiedliche
Gruppen auch mit unterschiedlichen Konsequenzen einhergehen: "It is too soon to tell what the final consequences will be, but it seems unlikely that they will ever be universal or stable“ (21). Im zweiten Kapitel betrachtet Baym das Zusammenspiel zwischen Technik und Mensch aus zwei Perspektiven - zunächst im Kontext des technischen Determinismus, der annimmt, dass "[...] technology is positioned as causing us and our social lives to change" (39). Anschließend behandelt sie eine sozialkonstruktivistische Perspektive, nach der sich die Nutzung digitaler Medien aus sozialen Kontexten heraus ergibt und demnach auch als eine Konsequenz menschlicher Ansprüche zu verstehen ist. Sozialer Wandel ist sodann keine Folge der Etablierung digitaler Medien, sondern vielmehr sind neue Technologien eine Schlussfolgerung sozialer Faktoren. Schließlich sieht Baym jedoch davon ab, sich einer dieser Positionen anzuschließen: "The truth, as is so often the case, lies somerwhere in between " (S. 44). Eine integrative dritte Perspektive ("social shaping of technology", S. 44) scheint deshalb sinnvoll, die auf die Interrelationen von Technologie und Sozialem abhebt: "Machines do not make history by themselves. But some kinds of machines help make different kinds of histories and different kinds of people than others" (Douglas 2004: 21).

Im dritten Kapitel beschäftigt sich Baym mit den Auswirkungen digitaler Medien auf die Kommunikation selbst. Besprochen wird entlang der populären „Reduced social Cues“-Annahme, welchen Einfluss das Fehlen sozialer Hinweisreize auf die Qualität der Kommunikation (z. B. flaming) haben kann, aber auch, wie dieser Mangel durch die digitale Kommunikation kompensiert werden kann (z. B. durch Emoticons und Akronyme). Die Autorin empfiehlt, digitale Kommunikation nicht den Ansprüchen von Face-to-Face-Kommunikation gegenüberzustellen, sondern diese vielmehr im Sinne einer "mixed modality“ (61) von Face-toFace- und textueller Kommunikation zu verstehen.

Das vierte Kapitel widmet sich der Analyse von Online Communities und ist für den aktuellen Gegenstandsbereich des Social Webs interessant, da hier nun explizit soziale OnlineNetzwerke (z. B. Facebook) angesprochen werden. Die beiden abschließenden Kapitel beschäftigen sich dann intensiver mit dyadischer Kommunikation: Im fünften Kapitel wird die häufig kontrovers diskutierte Frage angesprochen, ob von der Möglichkeit der Veröffentlichung von Falschinformationen über die eigene Person tatsächlich auch in der digitalen Kom- 